\title{
VIROPORIN ACTIVITY OF SARS-CoV E PROTEIN
}

\author{
Ying Liao, James P. Tam, and Ding X. Liu*
}

\section{INTRODUCTION}

Viroporins are integral membrane proteins encoded by viruses that contain a highly hydrophobic domain able to form an amphipathic $\alpha$-helix and tend to oligomerize to form a hydrophilic pore after insertion into cellular membranes. They affect the vesicle system of host cells, glycoprotein trafficking, and membrane permeability, leading to the promotion of viral particle release. ${ }^{1}$ In this study, we showed that SARS-CoV E protein could obviously enhance membrane permeability to hygromycin B (HB), a protein synthesis inhibitor, upon expression in mammalian cells. This activity was shown to be associated with the transmembrane domain of E protein.

\section{MATERIALS AND METHODS}

$H B$ assay and construction of plasmids: HeLa cells were transfected with appropriate plasmids, pretreated with different concentrations of HB (Sigma), and labeled with $\left[{ }^{35} \mathrm{~S}\right]$ methionine/cysteine (Amersham). Cells were incubated in the presence or absence of HB, harvested and lysed. Total proteins were immunoprecipitated with appropriate antibodies, and analyzed by SDS $15 \%$ polyacrylamide gel electrophoresis.

Plasmids pFlagE and pFlagN was constructed by cloning an EcoRV- and EcoRIdigested PCR fragments into EcoRV- and EcoRI-digested pFlag vector. The Flag-tag was fused to the $\mathrm{N}$-terminal end of the $\mathrm{E}$ protein. Mutations were introduced into the $\mathrm{E}$ gene by two rounds of PCR and confirmed by automated sequencing. The mutant constructs included in this study are summarized in Fig. 1.

\section{RESULTS}

\subsection{Alteration of Membrane Permeability by the Expression of E Protein}

To test if E protein can alter the membrane permeability of mammalian cells, the Flag-tagged $\mathrm{E}$ protein was expressed in $\mathrm{HeLa}$ cells and $\mathrm{HB}$ assay was performed.

\footnotetext{
*School of Biological Sciences, Nanyang Technological University, Singapore 637551, and Institute of Molecular and Cell Biology, Proteos, Singapore 138673189.
} 


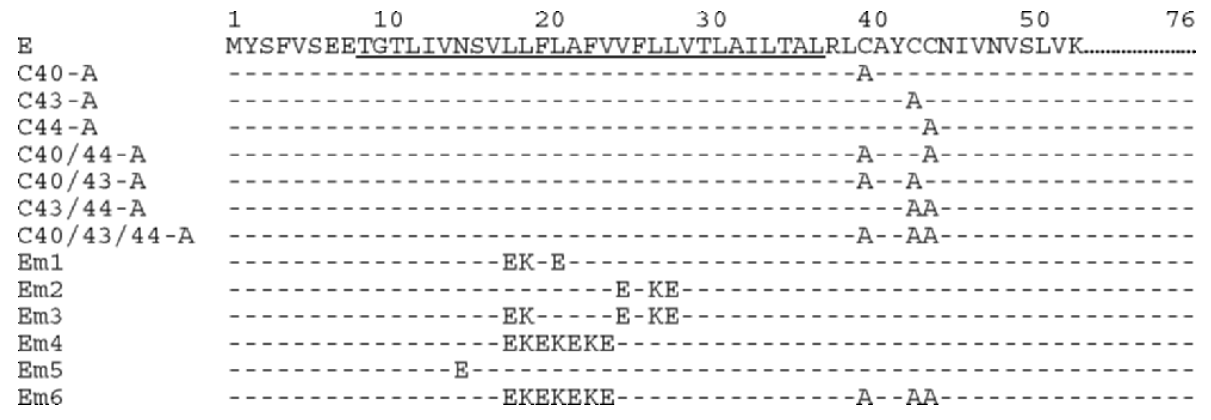

Figure 1. Amino acid sequence of wild-type and mutant SARS-CoV E protein. The putative transmembrane domain is underlined.

Extracts prepared from cells without treatment with $\mathrm{HB}$ showed detection of the $\mathrm{E}$ protein and some other cellular proteins (lane 1). In cells treated with 1 and $2 \mathrm{mM}$ of $\mathrm{HB}$, no obvious detection of the E protein and other cellular proteins was obtained (Fig. 2, lanes 2 and 3). However, as a negative control, in cells transfected with the SARS-CoV N protein, a similar amount of the $\mathrm{N}$ protein was detected in cells both treated and untreated with HB (Fig. 2, lanes 4-6).

\subsection{Mutational Analysis of the Three Cysteine Residues of E Protein}

SARS-CoV E protein contains three cysteine residues at amino acid positions 40, 43, and 44 , respectively. These residues are located 3-7 amino acids downstream of the transmembrane domain (Fig. 1). Mutations of these residues to alanine were made to generate seven mutants (C40-A, C43-A, C44-A, C43/44-A, C40/44-A, C40/43-A and C40/43/44-A) (Fig. 1). Western blot of cells expressing wild-type and most mutant constructs showed specific detection of three isoforms of $\mathrm{E}$ protein migrating at a range of molecular masses from 14 to $18 \mathrm{kDa}$ under reducing conditions (Fig. 3a). These isoforms may be derived from post-translational modifications of E protein.

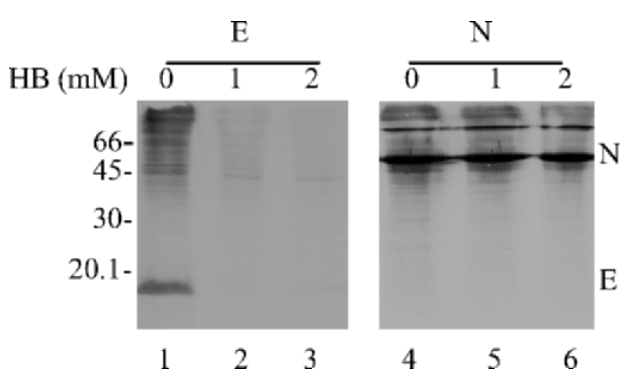

Figure 2. Modification of HeLa cells membrane permeability by SARS-CoV E protein. 


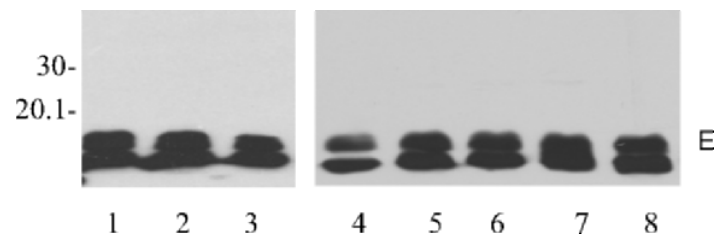

Figure 3a. Expression of wild-type (1), C40-A (2), C43-A (3), C44A (4), C43/44-A (5), C40/44-A (6), C40/43A (7) and C40/43/44-A (8) in HeLa cells.

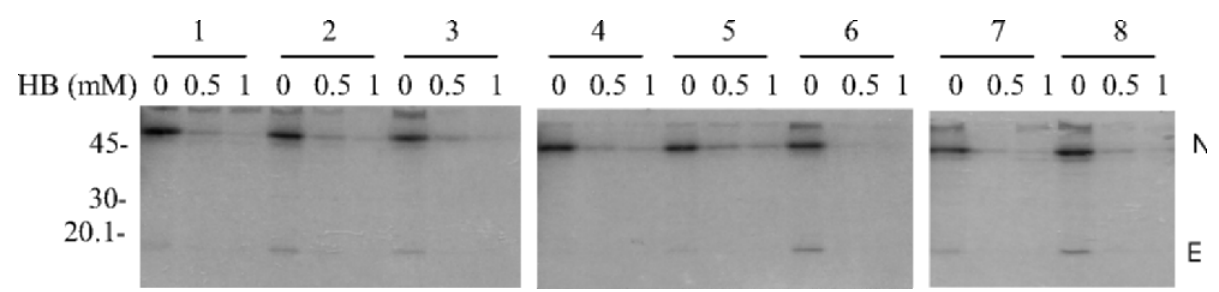

Figure 3b. Entry of Hygromycin B into HeLa cells expressing wild type and cysteine mutant $\mathrm{E}$ proteins. 1 $\mathrm{E}+\mathrm{N} ; 2: \mathrm{C} 40-\mathrm{A}+\mathrm{N} ; 3: \mathrm{C} 43-\mathrm{A}+\mathrm{N} ; 4: \mathrm{C} 44 \mathrm{~A}+\mathrm{N} ; 5: \mathrm{C} 43 / 44-\mathrm{A}+\mathrm{N} ; 6: \mathrm{C} 40 / 44-\mathrm{A}+\mathrm{N} ; 7: \mathrm{C} 40 / 43-\mathrm{A}+\mathrm{N} ; 8: \mathrm{C} 40 / 43 / 44-$ $\mathrm{A}+\mathrm{N}$.

In the membrane permeability assay shown in Fig. 3b, SARS-CoV N protein was cotransfected into HeLa cells together with wild-type and mutant E proteins. Expression of wild-type and mutant $\mathrm{E}$ protein showed that similar levels of inhibition of protein synthesis by HB were obtained (Fig. 3b).

\subsection{Mutational Analysis of the Transmembrane Domain of $E$ Protein}

SARS-CoV E protein contains a long putative transmembrane domain of 29 amino acid residues. ${ }^{2}$ This domain may be involved in the formation of ion channel by oligomerization. ${ }^{3}$ Mutations of the putative transmembrane domain were carried out to study its functional roles. Four mutants, Em1, Em2, Em3, and Em4, were made by mutation of 3-7 leucine/valine residues to charged amino acid residues in the transmembrane domain (Fig. 1). Em5 was constructed based on the molecular simulation studies showing that N15 residue may be essential for oligomerization of the protein (Fig. 1). Em6 was made by combination of Em4 and C40/43/44-A (Fig. 1). Expression of these mutants showed the detection of polypeptides with apparent molecular masses ranging from 10 to $18 \mathrm{kDa}$ (Fig. 4a). In the membrane permeability assay shown in Fig. 4b, cells expressing Em1, Em2, and Em5 exhibited a similar degree of inhibition of host protein synthesis as wild-type E protein (Fig 4b, lanes 1-9 and 16-18). In cells expressing Em3 and Em4, much less inhibition of protein synthesis by HB was observed compared with wild-type E protein (Fig. 4b, lanes 10-15). No inhibition of protein synthesis was observed in cells expressing Em6 (Fig. 4b, lanes 19-21). 
E Em1 Em2 Em3 Em4 Em5

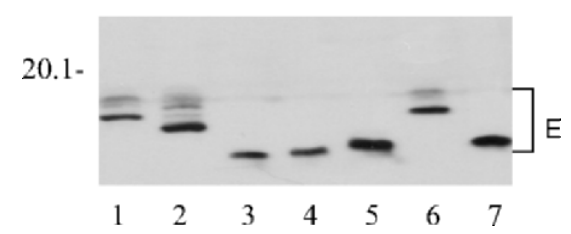

Figure 4a. Expression of the wild-type and transmembrane domain mutant E proteins in Hela cells.

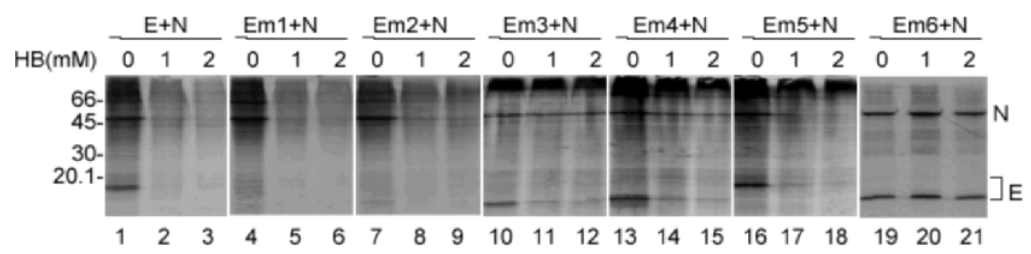

Figure 4b. Entry of Hygromycin B into Hela cells expressing wild-type and transmembrane domain mutant E proteins.

\section{DISCUSSION AND CONCLUSION}

In this study, we demonstrate that the membrane permeabilizing activity of SARS-CoV E protein is associated with the transmembrane domain. The C40 and C44 of the E protein were previously shown to play important roles in modification of membrane permeability in bacterial cells, ${ }^{4}$ but no obvious effect was observed in mammalian cells in this study. Only after combining mutation of the transmembrane domain and three cysteine residues was the membrane-permeabilizing activity of E protein totally disrupted. It suggests that these cysteine residues may play certain roles in the membrane association and membrane-permeabilizing activities of the $\mathrm{E}$ protein. A recent report that showed SAR-CoV E protein could form cation-selective ion channels further demonstrated $\mathrm{E}$ protein is a viroporin. ${ }^{5}$ It was also reported that MHV E protein could enhance membrane permeability in bacterial cells. ${ }^{6}$ These results suggest that E protein from different coronaviruses is endowed with viroporin activity. Disruption of the function of viroporins would abrogate viral infectivity, rendering E protein suitable targets for the development of antiviral drugs.

\section{REFERENCES}

1. Ciampor, F., 2003, The ion channels coded by viruses, Acta Microbiol. Immunol. Hung. 50:433-442.

2. Arbely, E., et al., 2004, A highly unusual palindromic transmembrane helical hairpin formed by SARS coronavirus E protein, J. Mol. Biol. 341:769-779.

3. Torres, J., et al., 2005, The transmembrane oligomers of coronavirus protein E, Biophys. J. 88:1283-1290.

4. Liao, Y., Lescar, J., Tam, J. P., and Liu, D. X., 2004, Expression of SARS-coronavirus envelope protein in Escherichia coli cells alters membrane permeability, Biochem. Biophys. Res. Commun. 325:374-380.

5. Wilson, L., McKinlay, C., Gage, P., and Ewart, G., 2004, SARS coronavirus E protein forms cation-selective ion channels, Virology 330:322-331.

6. Madan, V., et al., 2005, Viroporin activity of murine hepatitis virus E protein, FEBS Lett. 579:3607-3612. 